\title{
Corpus of Early American Literature ${ }^{1}$
}

\author{
Mikko Höglund, Stockholm University \\ Kaj Syrjänen, University of Tampere
}

\section{$1 \quad$ Introduction}

The use of electronic corpora has become widespread in many subfields of linguistics, and English, perhaps to some extent owing to its status as a lingua franca, has been the trailblazer in this methodology. The first widely used electronic corpus, the Brown corpus, compiled in the 1960s (see Kučera and Francis 1967), comprised American English texts, and as the field has advanced over the last decades, many of the pioneering corpora have consisted of English material. Nowadays, there is an abundance of English corpora available, including corpora of different national varieties of English, learner English, English for specific purposes, and historical English. However, even though the selection of corpora is impressive and broad, the basic subject matter and the object of linguistic research, the English language, is a veritable leviathan to investigate, and there are still notable gaps in the coverage of the available corpora.

In recent years and decades, many corpora have been compiled which document different periods in the history of English. To name a few, the Helsinki Corpus of English Texts is one of the first historical corpora, covering the period from the $8^{\text {th }}$ century to the $18^{\text {th }}$ century; the Corpus of Early English Correspondence (CEEC) and later versions of it comprise letters from 1403-1800; the Corpus of Late Modern English Texts (CLMET; De Smet 2005) and its successors CLMETEV and CLMET3.0 include texts from 1710-1920; and the Old Bailey Corpus (OBC) consists of transcripts of the proceedings of the Old Bailey criminal court from 1720 to 1913. In addition to the aforementioned corpora, which document English spoken and written in the Old World, there are also corpora that cover material from the other main variety, American English. The largest of these is the 400-million-word Corpus of Historical American English (COHA; Davies 2010-), which documents American English from 1810 to present day. Another corpus of historical American English is the Corpus of Early American English (Kytö 1994), which comprises texts from the period 1620-1720, and some historical corpora such as the Corpus of Late Mod- 
ern British and American English Prose (COLMOBAENG; 1700-1879) also include American English to some extent. We should also mention ARCHER ( $A$ Representative Corpus of Historical English Registers), a multi-genre corpus which covers a chronologically impressive time span from 1600 to 1999 of both British and American English.

Even though the entire time span of American English is fairly well documented, there is a noteworthy gap in the chronological coverage of the available corpora. The critical period for the formation of the AmE variety, from the early $18^{\text {th }}$ century before the declaration of independence and the times following the declaration, is not consistently documented. For instance, in COLMOBAENG there is some material from the $18^{\text {th }}$ century, and in COHA there is material from 1810 onwards, but there is no single corpus that would cohesively document that formative period. While ARCHER does cover this particular period, its total number of words for American English for the entire time span from 1600 to 1999 is only around 1.34 million (as of version 3.2).

The present paper introduces a corpus which is an endeavor to fill the existing gap in the selection of American English corpora. The corpus is called the Corpus of Early American Literature (CEAL) and it includes texts from 16901920. The corpus is divided into three subperiods, CEAL1 (1690-1780), CEAL2 (1781-1850), and CEAL3 (1851-1920), and the subperiods cover approximately 1.5, 5.7 and 6.3 million words, respectively (see Table 1). The compilation process and its challenges have been discussed in an earlier paper (Höglund and Syrjänen 2010) and will not be repeated in detail here. The aforementioned paper also outlines the historical events and circumstances that affected the formation of the new nation and with it the American English variety, and considers them in relation to the chronological subdivision of the corpus.

Table 1: Contents of CEAL in numbers

\begin{tabular}{lccc}
\hline Subcorpora & Number of texts (files) & Authors & Words \\
\hline CEAL1 (1690-1780) & 42 & 40 & $1,484,463$ \\
CEAL2 (1781-1850) & 65 & 56 & $5,740,042$ \\
CEAL3 (1851-1920) & 87 & 45 & $6,319,792$ \\
TOTAL & 194 & 141 & $13,544,297$ \\
\hline
\end{tabular}

The present paper is structured as follows: First, in Section 2 the structure and contents of CEAL are introduced together with a comprehensive list of the texts, 
publication years, authors and their birth and death years, and word counts. This is followed by a brief evaluation of the corpus in Section 3. Finally, Section 4 concludes the paper.

\section{Structure and contents of CEAL}

The compilation of CEAL was inspired by the CLMET, and the original idea was to create an American English counterpart of the British English CLMET for comparing linguistic phenomena in $\mathrm{BrE}$ and $\mathrm{AmE}$. The compilation criteria outlined in De Smet (2005) served as a starting point for CEAL; during the process of compiling CEAL these criteria were gradually modified to be more applicable for AmE. In its final form, CEAL is more or less comparable with the CLMET, but criteria-wise these corpora are not in their final form as similar to each other as e.g. LOB, BLOB, F-LOB and CLOB are to the Brown, B-Brown, Frown and Crown corpora.

The material for CEAL was retrieved from text repositories with open access and/or public domain material. The majority of the texts come from Project Gutenberg, with additional material from the American Studies Commons, Oxford Text Archive, and one text from the Bible Bulletin Board. The method used to collect the texts was very rudimentary and time-consuming, but also inclusive: all the English texts in the databases were manually browsed through, and suitable texts were then downloaded. While the bulk of the texts were available in plain text format, a handful of the texts only available in XML/ SGML or PDF were converted manually to plain text using freely available text conversion tools.

The text selection criteria were the following: the author of the text was born and educated on the new continent (or the author had a significant cultural/political influence there; see the discussion in Höglund and Syrjänen 2010: 439440 ), only full texts were included, and the word count per author was set to a maximum of 350,000 words. However, the total number of words for most of the authors in the corpus is less than 200,000. In addition, the relationship between the authors' birth year and the publication year(s) of their work was controlled in the same manner as in the CLMET (see Figure 1). This was done primarily to maximize the linguistic differences between the three subperiods. The effect of this method can be seen in Figure 2; most of the texts tend to concentrate towards the latter parts of the three subperiods. Also, in the figure texts whose publication spans multiple years have been marked according to their starting year of publication. Notably, each of the lines represents a publication year and not an individual author or a text, so the word count in this graph is occasionally higher than the per-author word limit of 350,000. 


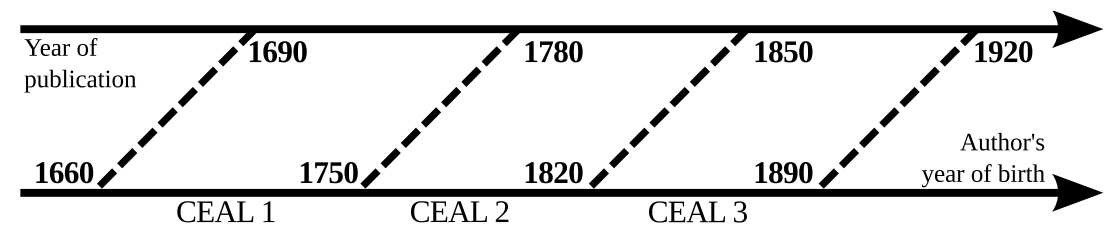

Figure 1: CEAL subperiods and publication year / author's birth year relationship

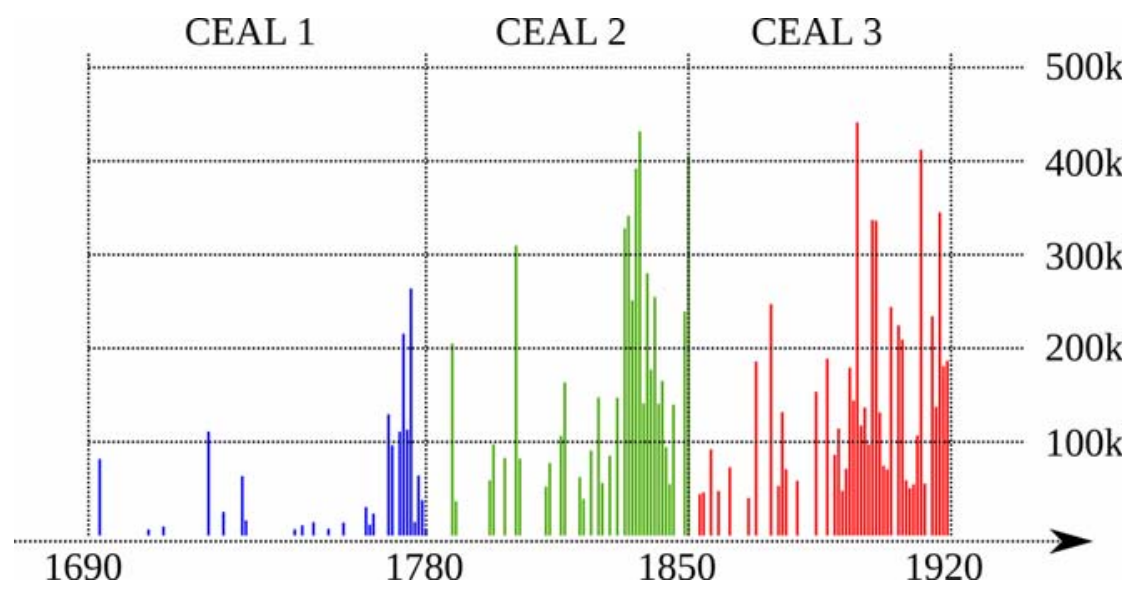

Figure 2: Chronological distribution of the texts in CEAL, with each line representing word count per publication year; texts whose publication spans multiple years are marked according to the starting year

The genre distribution between the three subperiods is somewhat uneven. The original aim was to collect mostly fiction texts in order to make CEAL as comparable with the CLMET as possible, but due to historical realities, it proved not to be possible. Whereas for CEAL2 and CEAL3 there is relatively much fictive material available in the text repositories, for the CEAL1 period, spanning between the late $17^{\text {th }}$ century and 1780 , there is not much fiction around that would be native to the American continent, as the compilation criteria stipulated. Apparently, the main problem is not so much the availability of the online material (although much less AmE material is available from the CEAL 1 period than the other periods), but the fact that, at that time, people on the new conti- 
nent were hardly writing any fiction of their own. Consequently, almost all of the texts currently included in CEAL1 are political writings, correspondence, journals, and religious texts. In CEAL2 the amount of fiction rises considerably; approximately 42 per cent of the texts are fictive prose. However, still more than half of the texts in CEAL2 are (auto)biographies, political and religious texts, journals, and other non-fiction texts. Lastly, CEAL3 consists almost solely of fictive texts; non-fiction covers only about 7 per cent of the subcorpus.

Regarding the timespan of CEAL2 and especially CEAL3, there was much material available in the text repositories, and we wanted to include many different styles of texts: colloquial style (e.g. David Crockett), complex style (e.g. Henry James), and texts that represent different vernaculars (e.g. F. Norris's McTeague; Grey's Riders of the Purple Sage), and texts by both male and female authors. The aim was to include as large a variety of styles as possible within the limits of the corpus. Also, if there were several books available by one author, we chose the author's early as well as late works.

The texts that are available in the online repositories from which the texts for CEAL were retrieved have not been uploaded with linguistic research in mind. This means that there is no guarantee that the texts follow the original spelling and form of the source text precisely. For some texts there are multiple versions available, with varying levels of accuracy; for instance, some digitized texts are accompanied by a transcriber's notes section showing the changes made to the original text during digitization. When compiling the corpus, whenever there was an opportunity to make a choice between two or more editions of the same work, we chose the one that we judged to be closest to the original (see the case of Franklin's letters below).

Some editing was done to the texts during the compilation process. For example, in some cases we included only parts of the larger collections of texts that were available by taking only the texts by a certain author from a larger work covering several writers. This was done, for instance, with The Diplomatic Correspondence of the American Revolution series of books, from which we only included correspondence by specific authors that fit the compilation criteria (e.g. from volume 7 only John Jay's correspondence was included). Since especially the first subcorpus includes a considerable number of small text sources with potentially overlapping passages (such as separate works covering letters from the same author), we also checked the subcorpora for duplicate texts or redundant passages using an automatic ad hoc approach, which involved replacing line breaks from each text with spaces and slicing the resulting text into 50character strings, each of which was searched from all the other texts in the subcorpus. This approach worked surprisingly well, helping us identify, for 
example, various letters by Benjamin Franklin that were included in two volumes of his writings (Memoirs vol. 2 and Letters) but edited in a different way. The duplicate texts were compared to pictures of Franklin's original writings found online, and we decided to keep the letters in Letters, as they seemed to be closer to the originals (for example, they retained Franklin's original spelling of the past participle: suppos'd, concern'd). Of course, this means that the rest of Memoirs, both volumes, which are more heavily edited, are still included in the corpus. Keeping this in mind, we would not recommend using the corpus for research on linguistic phenomena that are susceptible to editing, such as spelling, punctuation, and so forth. On the other hand, we see that other phenomena such as grammar, syntax, vocabulary and semantics are not as likely to have been altered by editors, and are better subjects of investigation using CEAL.

Table 2 lists the texts that are included in CEAL. The table also includes the publication year of each text, the names of the authors and their years of birth and death, and the word counts for both individual texts and authors. ${ }^{2}$

Table 2: Contents of CEAL

CEAL1 (1690-1780)

\begin{tabular}{|c|c|c|c|c|c|}
\hline Author & $\begin{array}{l}\text { Birth- } \\
\text { Death }\end{array}$ & Year & Title & $\begin{array}{l}\text { Word } \\
\text { count }\end{array}$ & $\begin{array}{l}\text { Words/ } \\
\text { author }\end{array}$ \\
\hline $\begin{array}{l}\text { Lyon, Lemuel; Haws, } \\
\text { Samuel }\end{array}$ & - & $1758-1775$ & $\begin{array}{l}\text { The Military Jour- } \\
\text { nals of two private } \\
\text { soldiers }\end{array}$ & 13816 & \\
\hline $\begin{array}{l}\text { Adams, John; Car- } \\
\text { michael, William; } \\
\text { Deane, Silas; Franklin, } \\
\text { Benjamin; Lee, Arthur; } \\
\text { Lovell, James; Morris, } \\
\text { Robert. }\end{array}$ & - & $1775-1780$ & $\begin{array}{l}\text { The Diplomatic } \\
\text { Correspondence of } \\
\text { the American Rev- } \\
\text { olution, Vol. } 9\end{array}$ & 16173 & \\
\hline $\begin{array}{l}\text { Deane, Silas; Franklin, } \\
\text { Benjamin; Harrison, } \\
\text { Benjamin; Hayward, } \\
\text { Thomas; Hooper, Will- } \\
\text { iam; Lee, Arthur; Lee, } \\
\text { Richard Henry; Living- } \\
\text { ston, Philip; Lovell, } \\
\text { James; Morris, Robert; } \\
\text { Whipple, William; } \\
\text { Witherspoon, John }\end{array}$ & - & $1776-1779$ & $\begin{array}{l}\text { The Diplomatic } \\
\text { Correspondence of } \\
\text { the American Rev- } \\
\text { olution, Vol. } 1\end{array}$ & 66818 & \\
\hline
\end{tabular}




\begin{tabular}{|c|c|c|c|c|c|}
\hline \multirow[t]{3}{*}{ Mather, Cotton } & \multirow[t]{3}{*}{$1663-1728$} & 1693 & $\begin{array}{l}\text { The wonders of the } \\
\text { invisible world }\end{array}$ & 81687 & \multirow[t]{3}{*}{98008} \\
\hline & & 1706 & $\begin{array}{l}\text { The Negro Chris- } \\
\text { tianized }\end{array}$ & 6527 & \\
\hline & & 1710 & $\begin{array}{l}\text { Theopolis Ameri- } \\
\text { cana }\end{array}$ & 9794 & \\
\hline Beverley, Robert Jr. & $1673-1722$ & 1722 & $\begin{array}{l}\text { The history of Vir- } \\
\text { ginia }\end{array}$ & 86808 & \\
\hline \multirow[t]{3}{*}{ Edwards, Jonathan } & $1703-1758$ & $1731-1750$ & $\begin{array}{l}\text { Selected Sermons } \\
\text { of Jonathan } \\
\text { Edwards }\end{array}$ & 63404 & \multirow[t]{3}{*}{90603} \\
\hline & & 1732 & Christian Charity & 16263 & \\
\hline & & 1747 & True Saints & 10936 & \\
\hline Chauncy, Charles & $1705-1787$ & 1745 & $\begin{array}{l}\text { Marvellous Things } \\
\text { done by the right } \\
\text { Hand and holy } \\
\text { Arm of God in get- } \\
\text { ting him the Vic- } \\
\text { tory }\end{array}$ & 6733 & \\
\hline \multirow[t]{4}{*}{ Franklin, Benjamin } & $1706-1790$ & $1722-1726$ & $\begin{array}{l}\text { Boston and Lon- } \\
\text { don - Writings } \\
1722-1726\end{array}$ & 24296 & \multirow[t]{4}{*}{145655} \\
\hline & & $1726-1757$ & Letters & 25107 & \\
\hline & & $1771-1790$ & Memoirs (1) & 35553 & \\
\hline & & $1771-1790$ & Memoirs (2) & 60699 & \\
\hline Mayhew, Jonathan & $1720-1766$ & 1750 & $\begin{array}{l}\text { A Discourse con- } \\
\text { cerning Unlimited } \\
\text { Submission and } \\
\text { Non-Resistance to } \\
\text { the Higher Powers }\end{array}$ & 14237 & \\
\hline Woolman, John & $1720-1772$ & 1774 & $\begin{array}{l}\text { The Journal of } \\
\text { John Woolman }\end{array}$ & 79276 & \\
\hline \multirow[t]{3}{*}{ Adams, Samuel } & $1722-1803$ & $1770-1773$ & $\begin{array}{l}\text { The Writings of } \\
\text { Samuel Adams (2) }\end{array}$ & 129423 & \multirow[t]{3}{*}{304475} \\
\hline & & $1773-1777$ & $\begin{array}{l}\text { The Writings of } \\
\text { Samuel Adams (3) }\end{array}$ & 111026 & \\
\hline & & $1778-1802$ & $\begin{array}{l}\text { The Writings of } \\
\text { Samuel Adams (4) }\end{array}$ & 64026 & \\
\hline
\end{tabular}




\begin{tabular}{|c|c|c|c|c|c|}
\hline \multirow[t]{2}{*}{ Otis, James Jr. } & \multirow[t]{2}{*}{$1725-1783$} & 1764 & $\begin{array}{l}\text { The Rights of Brit- } \\
\text { ish Colonies } \\
\text { Asserted and } \\
\text { Proved }\end{array}$ & 30527 & \multirow[t]{2}{*}{42316} \\
\hline & & 1765 & $\begin{array}{l}\text { A Vindication of } \\
\text { the British Colo- } \\
\text { nies }\end{array}$ & 11789 & \\
\hline Leacock, John & $1729-1802$ & 1776 & $\begin{array}{l}\text { The Fall of British } \\
\text { Tyranny }\end{array}$ & 22447 & \\
\hline Sherwood, Samuel & $1730-1783$ & 1776 & $\begin{array}{l}\text { The Church's } \\
\text { Flight into the Wil- } \\
\text { derness }\end{array}$ & 14432 & \\
\hline Stocking, Abner & $1730-1806$ & 1775 & $\begin{array}{l}\text { An interesting } \\
\text { journal of Abner } \\
\text { Stocking of } \\
\text { Chatham, Conneti- } \\
\text { cut }\end{array}$ & 9104 & \\
\hline Rogers, Robert & $1727-1795$ & 1766 & Ponteach & 23571 & \\
\hline Keteltas, Abraham & $1732-1798$ & 1777 & God Arising & 9823 & \\
\hline Washington, George & $1732-1799$ & 1754 & $\begin{array}{l}\text { The Journal of } \\
\text { Major George } \\
\text { Washington }\end{array}$ & 7284 & \\
\hline Noble, Oliver & $\begin{array}{l}1733 / 4- \\
1792\end{array}$ & 1775 & $\begin{array}{l}\text { Some Strictures } \\
\text { upon the Sacred } \\
\text { Story Recorded in } \\
\text { the Book of Esther }\end{array}$ & 11007 & \\
\hline Adams, John & $1735-1826$ & $1774-1780$ & $\begin{array}{l}\text { Familiar Letters of } \\
\text { John Adams and } \\
\text { His Wife Abigail } \\
\text { Adams, During the } \\
\text { Revolution }\end{array}$ & 80949 & \\
\hline Henry, Patrick & $1736-1799$ & 1775 & $\begin{array}{l}\text { Give Me Liberty or } \\
\text { Give Me Death }\end{array}$ & 1223 & \\
\hline Deane, Silas & $1737-1789$ & $1776-1779$ & $\begin{array}{l}\text { The Diplomatic } \\
\text { Correspondence of } \\
\text { the American Rev- } \\
\text { olution, Vol. } 1\end{array}$ & 56986 & \\
\hline \multirow[t]{2}{*}{ Paine, Thomas } & $1737-1809$ & 1776 & Common Sense & 21667 & 78681 \\
\hline & & $1776-1780$ & $\begin{array}{l}\text { The Writings of } \\
\text { Thomas Paine (1) }\end{array}$ & 57014 & \\
\hline
\end{tabular}




\begin{tabular}{|c|c|c|c|c|}
\hline Carmichael, William & $1739-1795$ & $1776-1780$ & $\begin{array}{l}\text { The Diplomatic } \\
\text { Correspondence of } \\
\text { the American Rev- } \\
\text { olution, Vol. } 9\end{array}$ & 15635 \\
\hline $\begin{array}{l}\text { Drayton, William- } \\
\text { Henry }\end{array}$ & $1742-1779$ & 1776 & $\begin{array}{l}\text { A Charge, on the } \\
\text { Rise of the Ameri- } \\
\text { can Empire }\end{array}$ & 8991 \\
\hline Jefferson, Thomas & $1743-1826$ & $1775-1780$ & $\begin{array}{l}\text { Memoirs, Corre- } \\
\text { spondences, and } \\
\text { Miscellanies, from } \\
\text { the Papers of Tho- } \\
\text { mas Jefferson }\end{array}$ & 75450 \\
\hline Adams, Abigail & $1744-1818$ & $1774-1780$ & $\begin{array}{l}\text { Familiar Letters of } \\
\text { John Adams and } \\
\text { His Wife Abigail } \\
\text { Adams, During the } \\
\text { Revolution }\end{array}$ & 55202 \\
\hline Jay, John & $1745-1829$ & $1779-1780$ & $\begin{array}{l}\text { The Diplomatic } \\
\text { Correspondence of } \\
\text { the American Rev- } \\
\text { olution, Vol. } 7\end{array}$ & 33372 \\
\hline Green, Ezra & $1746-1847$ & $1777-1778$ & $\begin{array}{l}\text { Diary of Ezra } \\
\text { Green }\end{array}$ & 4690 \\
\hline Hardenbergh, John L. & $1748-1806$ & 1779 & $\begin{array}{l}\text { The Journal of Lt. } \\
\text { John L. Harden- } \\
\text { bergh }\end{array}$ & 4486 \\
\hline Dodge, John & $1751-1800$ & 1780 & $\begin{array}{l}\text { The Dodge Narra- } \\
\text { tive }\end{array}$ & 6212 \\
\hline
\end{tabular}


CEAL2 (1781-1850)

\begin{tabular}{|c|c|c|c|c|c|}
\hline Author & $\begin{array}{l}\text { Birth- } \\
\text { Death }\end{array}$ & Year & Title & $\begin{array}{l}\text { Word } \\
\text { count }\end{array}$ & $\begin{array}{l}\text { Words/ } \\
\text { author }\end{array}$ \\
\hline $\begin{array}{l}\text { Hamilton, Alexander; } \\
\text { Madison, James }\end{array}$ & $\begin{array}{l}1755- \\
1804 ; \\
1751-1836\end{array}$ & $1787-1788$ & Federalist Papers & 183825 & \\
\hline Waterhouse, Benjamin & $1754-1846$ & 1816 & $\begin{array}{l}\text { A Journal of a } \\
\text { Young Man from } \\
\text { Massachusetts }\end{array}$ & 106372 & \\
\hline Biggs, William & $1755-1827$ & 1788 & $\begin{array}{l}\text { Indian Captivity of } \\
\text { William Biggs }\end{array}$ & 10673 & \\
\hline Marshall, John & $1755-1835$ & $1804-1807$ & $\begin{array}{l}\text { The Life of George } \\
\text { Washington }\end{array}$ & 121068 & \\
\hline Tyler, Royall & $1757-1826$ & 1787 & The Contrast & 21438 & \\
\hline $\begin{array}{l}\text { Webster Foster, } \\
\text { Hannah }\end{array}$ & $1758-1840$ & 1797 & The Coquette & 58999 & \\
\hline Monroe, James & $1758-1831$ & $1817-1824$ & $\begin{array}{l}\text { State of the Union } \\
\text { Address }\end{array}$ & 42400 & \\
\hline Weems, Parson & $1759-1825$ & 1805 & $\begin{array}{l}\text { Weems' Life of } \\
\text { General Francis } \\
\text { Marion }\end{array}$ & 82258 & \\
\hline $\begin{array}{l}\text { Daggett, David } \\
\text { (Jonathan Steadfast) }\end{array}$ & $1764-1851$ & 1804 & Count the Cost & 11009 & \\
\hline James, William Dobein & $1764-1830$ & 1821 & $\begin{array}{l}\text { A Sketch of the } \\
\text { Life of Brig. Gen. } \\
\text { Francis Marion }\end{array}$ & 62500 & \\
\hline Low, Samuel & $1765-?$ & 1788 & $\begin{array}{l}\text { The Politician Out- } \\
\text { Witted }\end{array}$ & 25753 & \\
\hline Dunlap, William & 1766-1839 & 1798 & André & 14846 & \\
\hline Jackson, Andrew & $1767-1845$ & $1829-1836$ & $\begin{array}{l}\text { State of the Union } \\
\text { Address }\end{array}$ & 85257 & \\
\hline $\begin{array}{l}\text { Harris, Thaddeus } \\
\text { Mason }\end{array}$ & $1768-1842$ & 1841 & $\begin{array}{l}\text { Biographical } \\
\text { Memorials of } \\
\text { James Oglethorpe }\end{array}$ & 96251 & \\
\hline \multirow[t]{2}{*}{$\begin{array}{l}\text { Brown, Charles } \\
\text { Brockden }\end{array}$} & $1771-1810$ & 1798 & $\begin{array}{l}\text { Wieland; or the } \\
\text { Transformation }\end{array}$ & 82682 & 165794 \\
\hline & & & Jane Talbot & 83112 & \\
\hline
\end{tabular}




\begin{tabular}{|c|c|c|c|c|c|}
\hline Dodge, David Low & $1774-1852$ & 1812 & $\begin{array}{l}\text { War Inconsistent } \\
\text { with the Religion } \\
\text { of Jesus Christ }\end{array}$ & 52289 & \\
\hline $\begin{array}{l}\text { Meriwether, Lewis; } \\
\text { Clark, William }\end{array}$ & $\begin{array}{l}1774- \\
1809 ; \\
1770-1838\end{array}$ & 1804 & $\begin{array}{l}\text { History of the } \\
\text { Expedition under } \\
\text { the Command of } \\
\text { Captains Lewis } \\
\text { and Clark, vol. } 1\end{array}$ & 177581 & \\
\hline $\begin{array}{l}\text { Tucker, George (Joseph } \\
\text { Atterley) }\end{array}$ & $1775-1861$ & 1827 & $\begin{array}{l}\text { Voyage to the } \\
\text { Moon }\end{array}$ & 55992 & \\
\hline Dunham, Jacob & $1779-?$ & 1850 & Journal of Voyages & 62688 & \\
\hline Webster, Daniel & $1782-1852$ & $1817-1845$ & $\begin{array}{l}\text { Select Speeches of } \\
\text { Daniel Webster }\end{array}$ & 121161 & \\
\hline Irving, Washington & $1783-1859$ & 1836 & Astoria & 161117 & \\
\hline Crockett, David & $1786-1836$ & 1834 & $\begin{array}{l}\text { Narrative of the } \\
\text { Life of David } \\
\text { Crockett }\end{array}$ & 53788 & \\
\hline Grandy, Moses & $1786 ?-?$ & 1842 & $\begin{array}{l}\text { Narrative of the } \\
\text { Life of Moses } \\
\text { Grandy }\end{array}$ & 13907 & \\
\hline \multirow[t]{3}{*}{$\begin{array}{l}\text { English, George } \\
\text { Bethune }\end{array}$} & $1787-1828$ & 1813 & $\begin{array}{l}\text { The Grounds of } \\
\text { Christianity }\end{array}$ & 77560 & 158026 \\
\hline & & 1822 & $\begin{array}{l}\text { A Narrative of the } \\
\text { Expedition to Don- } \\
\text { gola and Sennaar }\end{array}$ & 38938 & \\
\hline & & 1824 & $\begin{array}{l}\text { Five Pebbles from } \\
\text { the Brook }\end{array}$ & 41528 & \\
\hline Leslie, Eliza & $1787-1858$ & 1833 & Pencil Sketches & 173598 & \\
\hline Seaver, James E. & $1787-1827$ & 1824 & $\begin{array}{l}\text { A Narrative of the } \\
\text { Life of Mrs. Mary } \\
\text { Jemison }\end{array}$ & 49188 & \\
\hline \multirow[t]{2}{*}{$\begin{array}{l}\text { Cooper, James } \\
\text { Fenimore }\end{array}$} & $1789-1851$ & 1826 & $\begin{array}{l}\text { The Last of the } \\
\text { Mohicans }\end{array}$ & 147644 & 324934 \\
\hline & & 1840 & Pathfinder & 177290 & \\
\hline Fisk, Willbur & $1792-1839$ & 1835 & $\begin{array}{l}\text { Calvinistic Contro- } \\
\text { versy }\end{array}$ & 80798 & \\
\hline $\begin{array}{l}\text { Withers, Alexander } \\
\text { Scott }\end{array}$ & $1792-1865$ & 1831 & $\begin{array}{l}\text { Chronicles of Bor- } \\
\text { der Warfare }\end{array}$ & 147303 & \\
\hline
\end{tabular}




\begin{tabular}{|c|c|c|c|c|c|}
\hline $\begin{array}{l}\text { Goodrich, Samuel } \\
\text { Griswold }\end{array}$ & $1793-1860$ & 1844 & $\begin{array}{l}\text { Lives of Cele- } \\
\text { brated Women }\end{array}$ & 94337 & \\
\hline \multirow[t]{2}{*}{$\begin{array}{l}\text { Schoolcraft, Henry } \\
\text { Rowe }\end{array}$} & $1793-1864$ & 1839 & $\begin{array}{l}\text { Algic Researches } \\
\text { vol. } 1\end{array}$ & 53481 & 105856 \\
\hline & & 1839 & $\begin{array}{l}\text { Algic Researches } \\
\text { vol. } 2\end{array}$ & 52371 & \\
\hline Bryant, William Cullen & $1794-1878$ & $1834-1850$ & $\begin{array}{l}\text { Letters of a } \\
\text { Traveller }\end{array}$ & 105722 & \\
\hline \multirow[t]{2}{*}{ Drake, Benjamin } & $1795-1841$ & 1838 & $\begin{array}{l}\text { The Great Indian } \\
\text { Chief of the West }\end{array}$ & 71466 & 155896 \\
\hline & & 1841 & $\begin{array}{l}\text { Life of Tecumseh, } \\
\text { and of His Brother } \\
\text { the Prophet }\end{array}$ & 84430 & \\
\hline Kennedy, John P. & $1795-1870$ & 1838 & Rob of the Bowl & 69745 & \\
\hline Ames, Nathaniel & $1796-1835$ & 1835 & $\begin{array}{l}\text { An Old Sailor's } \\
\text { Yarns }\end{array}$ & 84541 & \\
\hline Prescott, William H. & $1796-1859$ & 1837 & $\begin{array}{l}\text { History of the } \\
\text { Reign of Ferdinand } \\
\text { and Isabella, the } \\
\text { Catholic, vol. } 1\end{array}$ & 152918 & \\
\hline Ware, William & $1797-1852$ & 1836 & Zenobia & 158593 & \\
\hline \multirow[t]{2}{*}{ Alcott, William Andrus } & $1798-1859$ & 1835 & $\begin{array}{l}\text { The Young Man's } \\
\text { Guide }\end{array}$ & 85939 & 158199 \\
\hline & & 1836 & The Young Mother & 72260 & \\
\hline $\begin{array}{l}\text { Beecher, Catherine } \\
\text { Esther }\end{array}$ & $1800-1878$ & 1842 & $\begin{array}{l}\text { A Treatise on } \\
\text { Domestic Econ- } \\
\text { omy }\end{array}$ & 126969 & \\
\hline Seward, William H. & $1801-1872$ & 1849 & $\begin{array}{l}\text { Life and Public } \\
\text { Services of John } \\
\text { Quincy Adams }\end{array}$ & 109257 & \\
\hline \multirow[t]{2}{*}{ Caruthers, William A. } & $1802-1846$ & 1834 & $\begin{array}{l}\text { The Cavaliers of } \\
\text { Virginia, vol. } 1\end{array}$ & 53637 & 112529 \\
\hline & & 1834 & $\begin{array}{l}\text { The Cavaliers of } \\
\text { Virginia, vol. } 2\end{array}$ & 58892 & \\
\hline Child, Lydia Maria & $1802-1880$ & 1833 & $\begin{array}{l}\text { An Appeal in } \\
\text { Favor of that Class } \\
\text { of Americans } \\
\text { Called Africans }\end{array}$ & 84883 & \\
\hline
\end{tabular}




\begin{tabular}{|c|c|c|c|c|c|}
\hline Emerson, Ralph Waldo & $1803-1882$ & $\begin{array}{l}1841 \\
1850\end{array}$ & $\begin{array}{l}\text { Essays, First Series } \\
\text { Representative } \\
\text { Men }\end{array}$ & $\begin{array}{l}74165 \\
57862\end{array}$ & 132027 \\
\hline Hawthorne, Nathaniel & 1804-1864 & 1837 & Twice-told Tales & 146175 & \\
\hline Bird, Robert M. & $1806-1854$ & 1837 & Nick of the Woods & 132653 & \\
\hline Spooner, Lysander & $1808-1887$ & 1845 & $\begin{array}{l}\text { The Unconstitu- } \\
\text { tionality of Slavery }\end{array}$ & 55065 & \\
\hline \multirow[t]{2}{*}{ Poe, Edgar Allan } & $1809-1849$ & $1833-44$ & $\begin{array}{l}\text { The Works of } \\
\text { Edgar Allan Poe- } \\
\text { Volume } 1\end{array}$ & 81434 & \multirow[t]{2}{*}{174249} \\
\hline & & $1834-49$ & $\begin{array}{l}\text { The Works of } \\
\text { Edgar Allan Poe- } \\
\text { Volume } 2\end{array}$ & 92815 & \\
\hline Ingraham, Joseph Holt & $1809-1860$ & $\begin{array}{l}1839 \\
1839\end{array}$ & $\begin{array}{l}\text { Captain Kyd, vol. } 1 \\
\text { Captain Kyd, vol. } 2\end{array}$ & $\begin{array}{l}69772 \\
69600\end{array}$ & 139372 \\
\hline Fuller, Margaret & $1810-1850$ & $1843-1850$ & $\begin{array}{l}\text { At Home and } \\
\text { Abroad }\end{array}$ & 164994 & \\
\hline Mayhew, Ira & 1814-1894 & 1850 & Popular Education & 145712 & \\
\hline Bibb, Henry & $1815-1854$ & 1849 & $\begin{array}{l}\text { Narrative of the } \\
\text { Life and Adven- } \\
\text { tures of Henry } \\
\text { Bibb, an American } \\
\text { Slave }\end{array}$ & 53062 & \\
\hline Halleck, Henry Wager & $1815-1872$ & 1846 & $\begin{array}{l}\text { Elements of Mili- } \\
\text { tary Art and Sci- } \\
\text { ence }\end{array}$ & 139888 & \\
\hline Eastman, Mary & $1818-1887$ & 1849 & Dacotah & 77094 & \\
\hline Melville, Herman & $1819-1891$ & 1850 & White-Jacket & 139497 & \\
\hline TOTAL & & & & 5740042 & \\
\hline
\end{tabular}


CEAL3 (1851-1920)

\begin{tabular}{|c|c|c|c|c|c|}
\hline Author & $\begin{array}{l}\text { Birth- } \\
\text { Death }\end{array}$ & Year & Title & $\begin{array}{l}\text { Word } \\
\text { count }\end{array}$ & $\begin{array}{l}\text { Words/ } \\
\text { author }\end{array}$ \\
\hline \multirow{4}{*}{$\begin{array}{l}\text { Adams, William Tay- } \\
\text { lor (Oliver Optic) }\end{array}$} & $1822-1897$ & 1854 & The Boat Club & 46104 & 233016 \\
\hline & & 1856 & Now or Never & 47660 & \\
\hline & & 1858 & Poor and Proud & 47669 & \\
\hline & & 1895 & Across India & 91583 & \\
\hline \multirow[t]{2}{*}{$\begin{array}{l}\text { Coffin, Charles } \\
\text { Carleton }\end{array}$} & $1823-1896$ & 1887 & $\begin{array}{l}\text { My Days and } \\
\text { Nights on the Bat- } \\
\text { tle-field }\end{array}$ & 65736 & 163939 \\
\hline & & 1895 & $\begin{array}{l}\text { Daughters of the } \\
\text { Revolution and } \\
\text { their Times }\end{array}$ & 98203 & \\
\hline \multirow{2}{*}{ Curtis, George William } & $1824-1892$ & 1853 & The Ptiphar Papers & 44273 & 88625 \\
\hline & & 1856 & Prue and I & 44352 & \\
\hline Warner, Charles & $1829-1900$ & 1872 & Backlog Studies & 52034 & 138242 \\
\hline Dudley & & 1889 & That Fortune & 86208 & \\
\hline Jackson, Helen Hunt & $1830-1885$ & 1884 & Ramona & 153588 & \\
\hline \multirow[t]{3}{*}{$\begin{array}{l}\text { Davis, Rebecca } \\
\text { Harding }\end{array}$} & $1831-1910$ & 1861 & $\begin{array}{l}\text { Life in the Iron- } \\
\text { Mills }\end{array}$ & 14707 & 109268 \\
\hline & & 1861 & Margret Howth & 58191 & \\
\hline & 1897 & & Frances Waldeaux & 36370 & \\
\hline \multirow[t]{2}{*}{ Perry, Nora } & $1831-1896$ & 1894 & Hope Benham & 64404 & 124772 \\
\hline & & 1895 & $\begin{array}{l}\text { A Flock of Girls } \\
\text { and Boys }\end{array}$ & 60368 & \\
\hline Alcott, Louisa May & $1832-1888$ & $1868-1869$ & Little Women & 186064 & \\
\hline \multirow[t]{2}{*}{ Alger, Horatio Jr. } & $1832-1899$ & 1866 & $\begin{array}{l}\text { Timothy Crump's } \\
\text { Ward }\end{array}$ & 40273 & 85532 \\
\hline & & 1887 & The Store Boy & 45259 & \\
\hline \multirow[t]{2}{*}{$\begin{array}{l}\text { Talmage, Thomas De } \\
\text { Witt }\end{array}$} & $1832-1902$ & 1872 & $\begin{array}{l}\text { The Abominations } \\
\text { of Modern Society }\end{array}$ & 53106 & 140140 \\
\hline & & 1895 & $\begin{array}{l}\text { Around the Tea- } \\
\text { Table }\end{array}$ & 87034 & \\
\hline $\begin{array}{l}\text { Twain, Mark (Samuel } \\
\text { Clemens) }\end{array}$ & $1835-1910$ & 1876 & $\begin{array}{l}\text { The Adventures of } \\
\text { Tom Sawyer }\end{array}$ & 70800 & \\
\hline Roe, Edward Payson & $1838-1888$ & 1872 & $\begin{array}{l}\text { Barriers Burned } \\
\text { Away }\end{array}$ & 142130 & \\
\hline
\end{tabular}




\begin{tabular}{|c|c|c|c|c|c|}
\hline \multirow[t]{2}{*}{$\begin{array}{l}\text { Bierce, Ambroce } \\
\text { Gwinnett }\end{array}$} & \multirow[t]{2}{*}{$1842-1914$} & 1874 & $\begin{array}{l}\text { Cobwebs from an } \\
\text { Empty Skull }\end{array}$ & 52941 & \multirow[t]{2}{*}{113005} \\
\hline & & 1893 & $\begin{array}{l}\text { Can Such Things } \\
\text { Be? }\end{array}$ & 60064 & \\
\hline \multirow[t]{2}{*}{ James, Henry } & \multirow[t]{2}{*}{ 1843-1916 } & 1875 & Roderick Hudson & 131723 & \multirow[t]{2}{*}{186074} \\
\hline & & 1911 & The Outcry & 54351 & \\
\hline \multirow[t]{3}{*}{ Jewett, Sarah Orne } & \multirow[t]{3}{*}{$1849-1909$} & 1879 & $\begin{array}{l}\text { Old Friends and } \\
\text { New }\end{array}$ & 58356 & \multirow[t]{3}{*}{157031} \\
\hline & & 1890 & Betty Leicester & 56619 & \\
\hline & & 1896 & $\begin{array}{l}\text { The Country of the } \\
\text { Pointed Firs }\end{array}$ & 42056 & \\
\hline \multirow[t]{2}{*}{ Bellamy, Edward } & \multirow[t]{2}{*}{$1850-1898$} & 1887 & Looking Backward & 77836 & \multirow[t]{2}{*}{183435} \\
\hline & & 1900 & $\begin{array}{l}\text { The Duke of } \\
\text { Stockbridge }\end{array}$ & 105599 & \\
\hline \multirow[t]{2}{*}{ Chopin, Kate } & \multirow[t]{2}{*}{$1850-1904$} & 1890 & At Fault & 57569 & \multirow[t]{2}{*}{121562} \\
\hline & & 1893-1899 & $\begin{array}{l}\text { The Awakening } \\
\text { and Selected Short } \\
\text { Stories }\end{array}$ & 63993 & \\
\hline \multirow[t]{2}{*}{ Marden, Orison Swett } & \multirow[t]{2}{*}{$1850-1924$} & $\begin{array}{l}1896 \\
1897\end{array}$ & $\begin{array}{l}\text { How to Succeed } \\
\text { Architects of Fate }\end{array}$ & $\begin{array}{l}75355 \\
100423\end{array}$ & \multirow[t]{2}{*}{246016} \\
\hline & & 1916 & $\begin{array}{l}\text { The Victorious } \\
\text { Attitude }\end{array}$ & 70238 & \\
\hline \multirow{2}{*}{$\begin{array}{l}\text { Freeman, Mary E. } \\
\text { Wilkins }\end{array}$} & \multirow[t]{2}{*}{$1852-1930$} & 1894 & Pembroke & 79906 & \multirow[t]{2}{*}{154221} \\
\hline & & 1900 & $\begin{array}{l}\text { The Heart's High- } \\
\text { way }\end{array}$ & 74315 & \\
\hline \multirow[t]{3}{*}{ van Dyke, Henry } & \multirow[t]{3}{*}{$1852-1933$} & 1895 & Little Rivers & 57968 & \multirow[t]{3}{*}{169603} \\
\hline & & 1901 & The Ruling Passion & 54865 & \\
\hline & & 1907 & Days Off & 56770 & \\
\hline \multirow[t]{2}{*}{ Rathborne, St. George } & \multirow[t]{2}{*}{$1854-1938$} & $\begin{array}{l}1893 \\
1912\end{array}$ & $\begin{array}{l}\text { Miss Caprice } \\
\text { Canoe Mates in } \\
\text { Canada }\end{array}$ & $\begin{array}{l}55230 \\
48456\end{array}$ & \multirow[t]{2}{*}{151409} \\
\hline & & 1912 & Chums in Dixie & 47723 & \\
\hline \multirow[t]{2}{*}{ Washington, Booker T. } & \multirow[t]{2}{*}{$1856-1915$} & 1899 & $\begin{array}{l}\text { The Future of the } \\
\text { American Negro }\end{array}$ & 38334 & \multirow[t]{2}{*}{114961} \\
\hline & & 1901 & $\begin{array}{l}\text { Up from Slavery: } \\
\text { an Autobiography }\end{array}$ & 76627 & \\
\hline
\end{tabular}




\begin{tabular}{|c|c|c|c|c|c|}
\hline $\begin{array}{l}\text { Gilman, Charlotte } \\
\text { Perkins }\end{array}$ & $1860-1935$ & $\begin{array}{l}1911 \\
1915\end{array}$ & $\begin{array}{l}\text { The Crux } \\
\text { Herland }\end{array}$ & $\begin{array}{l}52556 \\
52145\end{array}$ & 104701 \\
\hline Ottolengui, Rodrigues & $1861-1937$ & $\begin{array}{l}1892 \\
1898\end{array}$ & $\begin{array}{l}\text { An Artist in Crime } \\
\text { Final Proof }\end{array}$ & $\begin{array}{l}71380 \\
97466\end{array}$ & 168846 \\
\hline $\begin{array}{l}\text { Porter, William Syd- } \\
\text { ney (O. Henry) }\end{array}$ & $1862-1910$ & $\begin{array}{l}1904 \\
1907\end{array}$ & $\begin{array}{l}\text { Cabbages and } \\
\text { Kings } \\
\text { Heart of the West }\end{array}$ & $\begin{array}{l}63099 \\
77807\end{array}$ & 140906 \\
\hline Stratton-Porter, Gene & $1863-1924$ & 1915 & $\begin{array}{l}\text { Michael } \\
\text { O’Halloran }\end{array}$ & 145728 & \\
\hline \multirow[t]{4}{*}{ Davis, Richard Harding } & $1864-1916$ & 1891 & $\begin{array}{l}\text { Gallegher and } \\
\text { Other Stories }\end{array}$ & 47779 & 189553 \\
\hline & & 1902 & Ranson's Folly & 74271 & \\
\hline & & 1910 & Once upon a Time & 54586 & \\
\hline & & 1916 & $\begin{array}{l}\text { The Man that } \\
\text { Could Not Lose }\end{array}$ & 12917 & \\
\hline Phillips, David Graham & $1867-1911$ & $\begin{array}{l}1904 \\
1912\end{array}$ & $\begin{array}{l}\text { The Cost } \\
\text { The Price She Paid }\end{array}$ & $\begin{array}{l}75487 \\
96678\end{array}$ & 172165 \\
\hline \multirow[t]{2}{*}{ Porter, Eleanor H. } & $1868-1920$ & 1908 & $\begin{array}{l}\text { The Turn of the } \\
\text { Tide }\end{array}$ & 54332 & 113376 \\
\hline & & 1916 & Just David & 59044 & \\
\hline Tarkington, Booth & $1869-1946$ & 1918 & $\begin{array}{l}\text { The Magnificent } \\
\text { Ambersons }\end{array}$ & 99511 & \\
\hline \multirow[t]{2}{*}{ Norris, Frank } & $1870-1902$ & 1899 & McTeague & 112623 & 162922 \\
\hline & & 1903 & A Deal in Wheat & 48832 & \\
\hline \multirow[t]{3}{*}{ Rice, Alice Hegan } & $1870-1942$ & 1903 & Lovey Mary & 21758 & 161455 \\
\hline & & 1909 & Mr. Opp & 50465 & \\
\hline & & 1917 & Calvary Alley & 89119 & \\
\hline Churchill, Winston & $1871-1947$ & 1917 & $\begin{array}{l}\text { The Dwelling- } \\
\text { Place of Light }\end{array}$ & 138569 & \\
\hline \multirow[t]{3}{*}{ Crane, Stephen } & $1871-1900$ & 1895 & $\begin{array}{l}\text { The Red Badge of } \\
\text { Courage }\end{array}$ & 46203 & 161689 \\
\hline & & 1899 & Active Service & 79739 & \\
\hline & & 1899 & $\begin{array}{l}\text { The Monster and } \\
\text { Other Stories }\end{array}$ & 35747 & \\
\hline Dreiser, Theodore & $1871-1945$ & 1900 & Sister Carrie & 156282 & \\
\hline
\end{tabular}




\begin{tabular}{|c|c|c|c|c|c|}
\hline \multirow[t]{2}{*}{ MacGrath, Harold } & \multirow[t]{2}{*}{$1871-1932$} & 1899 & $\begin{array}{l}\text { Arms and the } \\
\text { Woman }\end{array}$ & 70704 & \multirow[t]{2}{*}{107107} \\
\hline & & 1915 & $\begin{array}{l}\text { The Voice in the } \\
\text { Fog }\end{array}$ & 36403 & \\
\hline \multirow[t]{2}{*}{ Grey, Zane } & \multirow[t]{2}{*}{$1872-1939$} & 1906 & $\begin{array}{l}\text { The Spirit of the } \\
\text { Border }\end{array}$ & 88039 & \multirow[t]{2}{*}{191736} \\
\hline & & 1912 & $\begin{array}{l}\text { Riders of the Pur- } \\
\text { ple Sage }\end{array}$ & 103697 & \\
\hline \multirow[t]{2}{*}{ Wright, Harold Bell } & \multirow[t]{2}{*}{$1872-1944$} & 1907 & $\begin{array}{l}\text { The Shepherd of } \\
\text { the Hills }\end{array}$ & 74842 & \multirow[t]{2}{*}{142190} \\
\hline & & 1919 & $\begin{array}{l}\text { The Re-Creation of } \\
\text { Brian Kent }\end{array}$ & 67348 & \\
\hline \multirow[t]{2}{*}{ Cather, Willa } & \multirow[t]{2}{*}{ 1873-1947 } & 1913 & O Pioneers! & 55435 & \multirow[t]{2}{*}{136755} \\
\hline & & 1918 & My Antonia & 81320 & \\
\hline Palmer, Frederick & $1873-1958$ & 1912 & Over the Pass & 115400 & \\
\hline \multirow[t]{2}{*}{ London, Jack } & \multirow[t]{2}{*}{$1876-1916$} & 1904 & The Sea-Wolf & 105651 & \multirow[t]{2}{*}{177715} \\
\hline & & 1906 & White Fang & 72064 & \\
\hline \multirow[t]{2}{*}{ Rinehart, Mary Roberts } & \multirow[t]{2}{*}{$1876-1958$} & 1906 & $\begin{array}{l}\text { The Man in Lower } \\
\text { Ten }\end{array}$ & 64452 & \multirow[t]{2}{*}{183728} \\
\hline & & 1919 & Dangerous Days & 119276 & \\
\hline Norris, Kathleen & $1880-1966$ & 1917 & $\begin{array}{l}\text { Martie the Uncon- } \\
\text { quered }\end{array}$ & 117605 & \\
\hline
\end{tabular}

TOTAL

6319792

\section{Evaluation}

In this section we will briefly assess CEAL in terms of its contents and, to some extent, usefulness for linguistic research. One challenge in the evaluation of CEAL is that there is no comparable corpus that could act as a point of reference. This means that, in order to evaluate the quality of CEAL, we can either compare it to corpora from other time periods and varieties (Section 3.1), explore its internal composition by cross-comparing the subcorpora (Section 3.2) or use it to study different phenomena in language and thereby indirectly judge its usefulness (Section 3.3). As the present paper is not a research paper but an introductory article to the corpus, the evaluations presented below are fairly straightforward. 


\subsection{Comparison with other corpora}

Evaluating the overall representativeness of CEAL in relation to other corpora is not without difficulties. If the CEAL data looks different from the other corpora, it might be either (a) because AmE 1690-1920 is simply different, or (b) because CEAL is unbalanced and/or biased. In order to minimize this issue when comparing with other corpora, we use corpora that are as close as possible to CEAL time- and variety-wise, and/or parameters that have been observed to remain more or less stable across time periods and varieties. The concordancer that was used for the searches was the freely available AntConc (Anthony 2014).

First, the most frequent words in CEAL were compared with the most frequent words in the CLMETEV, and the Brown and Frown corpora. The CLMETEV was chosen because it covers the same time span as CEAL, and Brown and Frown were chosen because they represent American English. The results are presented in Table 3:

Table 3: Twenty most frequent words in CEAL, the CLMETEV, and Brown + Frown

\begin{tabular}{lcccccc}
\hline & \multicolumn{2}{c}{ CEAL } & \multicolumn{2}{c}{ CLMETEV } & \multicolumn{2}{c}{ Brown + Frown } \\
rank & word & freq. pmw & word & freq. pmw & word & freq. pmw \\
\hline 1 & the & 64545 & the & 59588 & the & 66186 \\
2 & of & 35847 & of & 35863 & of & 34374 \\
3 & and & 33202 & and & 33068 & and & 28471 \\
4 & to & 28518 & to & 28845 & to & 26220 \\
5 & a & 21654 & a & 20958 & a & 22605 \\
6 & in & 17896 & in & 17823 & in & 20898 \\
7 & i & 13337 & i & 14883 & that & 11411 \\
8 & that & 12804 & that & 12669 & is & 9721 \\
9 & he & 11368 & it & 11686 & for & 9366 \\
10 & it & 11248 & was & 10801 & was & 8987 \\
11 & was & 10919 & he & 10615 & he & 8787 \\
12 & his & 9197 & his & 8870 & it & 8648 \\
13 & with & 8275 & as & 8358 & as & 7323 \\
14 & as & 7931 & is & 8244 & with & 7163 \\
15 & you & 7677 & with & 8205 & on & 6833
\end{tabular}




\begin{tabular}{lcccccc}
16 & for & 7589 & for & 7987 & his & 6379 \\
17 & is & 7300 & you & 7195 & be & 5785 \\
18 & had & 6618 & not & 6894 & by & 5252 \\
19 & her & 6433 & be & 6833 & at & 5113 \\
20 & be & 6410 & had & 6564 & i & 4828 \\
\hline
\end{tabular}

As can be seen in Table 3, the lists are generally very similar, sharing most of the words, and with the six most frequent tokens given in the same order. The proportions seem to be similar as well. Of course it has to be borne in mind that Brown and Frown include a variety of text types, while CEAL and the CLMETEV mostly cover fiction, personal letters and so forth, which, for instance, explains the relatively high frequency of the first person singular pronoun $I$ in them. Nevertheless, these results indicate that CEAL, as a whole, consists of what might be referred to as 'typical' language, without any major divergences.

\subsection{Comparison across the subcorpora}

In addition to the overall word frequency, we wanted to see the situation in the three subcorpora. The wordlists from CEAL1, CEAL2 and CEAL3 are presented in Table 4:

Table 4: most frequent words in CEAL subcorpora

\begin{tabular}{lccc}
\hline rank & CEAL1 & CEAL2 & CEAL3 \\
\hline 1 & the & the & the \\
2 & of & of & and \\
3 & and & and & to \\
4 & to & to & of \\
5 & in & in & a \\
6 & a & that & i \\
7 & that & it & in \\
8 & i & was & he \\
9 & it & i & was \\
10 & be & his & that \\
11 & is & with & it \\
12 & as & & you
\end{tabular}




\begin{tabular}{llll}
13 & for & is & his \\
14 & have & as & her \\
15 & with & he & she \\
16 & this & for & had \\
17 & by & be & with \\
18 & they & by & for \\
19 & their & which & as \\
20 & not & on & but \\
\hline
\end{tabular}

Table 4 displays more variation in the top-20 lists of words than Table 3, which might be expected, considering the different proportions of fiction and non-fiction texts in the three parts of the corpus. Nevertheless, the lists feature mostly the same words as the lists in Table 3, which is again seen as a sign of what might be considered 'typical' language.

\subsection{Practical application}

The corpus has already been piloted in linguistic research; Höglund (2014) used the work-in-progress version of CEAL in his doctoral dissertation to study the development of the tough construction (John is easy to please) in the past three centuries. In Höglund's study, CEAL1, with its modest size of $1.5 \mathrm{M}$ words, proved to be too small to investigate the fairly infrequent tough construction, but the larger CEAL2 and CEAL3 provided enough material for the research. The CEAL data was compared to the data from the CLMETEV, and the comparison showed quite nicely, for instance, that the tough construction began to be used more first in BrE around the early $19^{\text {th }}$ century, and that AmE followed the trend in the late $19^{\text {th }}$ century. In addition, the passive tough construction (John is easy to be pleased) was observed to follow the same pattern but in the other direction: it was falling into disfavor earlier in BrE than in AmE (Höglund 2014: 76-77). This type of change seems to be in accordance with earlier observations about 'colonial lag' (see Rohdenburg and Schlüter (2009), especially chapters 1 by Hundt, 5 by Schlüter, and 19 by Rohdenburg and Schlüter), and would thus validate the reliability of the results and indicate that CEAL represents AmE quite well at least in this respect. However, more studies of different types are of course needed in order to properly evaluate the usefulness of CEAL as a linguistic resource. 


\section{Conclusion}

The present paper has introduced a new resource for linguistic research that has not been previously available: the Corpus of Early American Literature (CEAL). Before CEAL, corpus material from the $18^{\text {th }}$ century written on the new continent was hardly available. The corpus covers a little over 200 years of texts, a period that includes the formation in the $18^{\text {th }}$ century of the variety now known as American English, as well as its further development in the $20^{\text {th }}$ century. That is, CEAL makes it possible to trace the development and formation of American English as a variety in its own right. Another advantage of CEAL is that the data can be fairly easily compared to the CLMETEV, which includes British English, as the two corpora are more or less comparable in terms of timespan, size, and compilation criteria.

It is our belief that the vast amount of text material that has been released through online repositories and endeavors for open access is massively beneficial for the study of English, which continuously needs larger and more specialized corpora with which to shed light on linguistic phenomena. All the material made available online has also made the compilation of corpora such as CEAL more accessible for linguists in general. In its current form, CEAL remains a fairly modest corpus when it comes to size, but one which helps form a more complete picture of the history and development of American English.

\section{Availability and disclaimer}

The corpus introduced in this article, CEAL, can be compiled by anyone by using the instructions and the text repositories mentioned, and downloading the texts in the content list. CEAL can also be obtained more easily by contacting one of the compilers. The usual disclaimers apply: the compilers are not responsible for the accuracy of the individual texts, and it is solely the responsibility of the user of the corpus to evaluate the authenticity of the texts and the suitability of the corpus for her/his research.

\section{Notes}

1. The project was related to and a part of Höglund's doctoral thesis (2014), which was funded by the Finnish Academy of Science and Letters, Jutikkala Fund. The compilers of the corpus are grateful for the comments received from Maarit Piipponen, David Robertson and Markku Salmela, of the University of Tampere, on the contents of the corpus. The final deci- 
sions on what to include in the corpus, and all the possible mistakes therein, are solely the authors' responsibility.

2. A more detailed table with notes on the texts and sources is available from the compilers.

\section{References}

Anthony, Laurence. 2014. AntConc (Version 3.4.4) [Computer Software]. Tokyo, Japan: Waseda University. Available from http://www.laurenceanthony.net/.

Davies, Mark. 2010-. The Corpus of Historical American English: 400 million words, 1810-2009. Available online at http://corpus.byu.edu/coha/.

De Smet, Hendrik. 2005. A Corpus of Late Modern English. ICAME Journal 29: 69-82.

Höglund, Mikko. 2014. "Self-discipline strategies were easy to design but difficult to adhere to." A usage-based study of the Tough Construction in English. Doctoral dissertation, University of Tampere.

Höglund, Mikko and Kaj Syrjänen. 2010. Towards a corpus of early American literature: On the challenges of compiling a comparable diachronic corpus. In I. Moskowich-Spiegel Fandiño, B. Crespo García, I. Lareo Martín and P. Lojo Sandino (eds.). Language windowing through corpora, 429-442. A Coruña: Universidade da Coruña.

Kučera, Henry and W. Nelson Francis. 1967. Computational analysis of Present-day American English. Providence, RI: Brown University Press.

Kytö, Merja. 1994. Towards a corpus of early American English. In M. Kytö, M. Rissanen and S. Wright (eds.). Corpora across the centuries, 33-39. Amsterdam and Atlanta: Rodopi.

Rohdenburg, Günter and Julia Schlüter (eds.). 2009. One language, two grammars? Differences between British and American English. Cambridge: Cambridge University Press. 